\title{
Quality and safety in acute medical care - what can design bring to the table?
}

\author{
${ }^{1}$ E Matthews \\ ${ }^{1}$ Reader in Healthcare Design, The Helen Hamlyn Centre for Design, Royal College of Art, London, UK
}

KEYWORDS acute medical care, co-design, design, inclusive design, people-centred design, user research

DECLARATION OF INTERESTS No conflict of interest declared

\author{
Correspondence to E Matthews \\ Patient Safety and Healthcare \\ The Helen Hamlyn Centre for Design \\ Royal College of Art \\ Kensington Gore \\ London SW7 2EU \\ UK
}

e-mail ed.matthews@rca.ac.uk
Acute hospital services are under unprecedented pressure. Complicating factors such as the ageing demographic and emerging epidemics of obesity, diabetes, respiratory disease and dementia, mean that as a society we can no longer hope to deliver the quality of healthcare that we would like to, and our systems are ceasing to be fit for purpose. Simply increasing efficiency and making incremental cost reductions are not going to fix these problems, and new thinking is required. The drive for integrated health and social care, and the Royal College of Physicians of London's Future Hospital report are good initiatives, but will current hospital environments, incrementally improved, actually be able to satisfy the demands we will make on them? Perhaps, given that something disruptive is needed, it might be valuable to think about what design might bring to the table.

Properly focused design can deliver innovative, systemsbased solutions that have been validated by healthcare practitioners, harnessing their experience, skills and creativity through a process of co-design, development and evaluation. The 'Double Diamond' development model,' in conjunction with Inclusive Design principles, ${ }^{2}$ builds on immersive research with users of a system. A full understanding of an identified problem allows the distillation of an evidence-based brief, outlining the sort of design intervention that needs to be developed to address it. System users and designers can co-design to generate a large number of creative ideas as potential conceptual solutions, and shortlist them for a process of iterative development, prototyping and evaluation. Hence, tested solutions can be piloted with the engagement and support of key stakeholders. ${ }^{3}$

First, let us consider what the terms 'Design', and in particular, 'Inclusive Design' mean.

'Design' is a very broad term with many definitions, not least because it may be applied to an object, a process or a system. 'Designs' are invariably communicated by some form of drawing, text specifications, numerical data, a physical master object or some combination of these. The layperson tends to associate 'design' and 'designer' with physical artefacts magically imbued with superior attributes relative to other 'non-designer' stuff. This may be as a result of a design approach that is conceptually different to the norm or because it has an interesting or special appearance that implies wealth, intelligence or out-of-the-ordinariness on the part of its owner. Indeed, in recent decades, the word 'designer' has been appropriated as an adjective to imply 'cool' or 'stylish' (or, perhaps, 'parts fools from their money'). This is not to imply that fashion should be completely dismissed; a life devoid of fun, aspiration, elegance and evolving styles would be dismal.

In my own experience of 35 years practising as a designer and engineer, designers themselves mostly seem to think of their discipline as a practical one that seeks to provide more or less creative solutions to problems, needs, or desires. There are areas where design contributes, at a fundamental level, in response to the most serious societal challenges: for example, increasing access to better products and services for a wider range of people regardless of age or disabilities. This also applies to healthcare: improving safety, dignity, efficiency and sustainability in the face of the ageing demographic; seeking solutions to challenges such as obesity, diabetes, dementia, chronic obstructive pulmonary disease; and wrestling with the dilemmas relating to the delivery of technology-enabled, costly treatments in a society that cannot generate the wealth to deliver them.

Next, what are the characteristics of 'acute medical care'? Straight out of Wikipedia, but broadly in line with the way a non-specialist 'designer' understands the term, acute medical care is the early and specialist management 


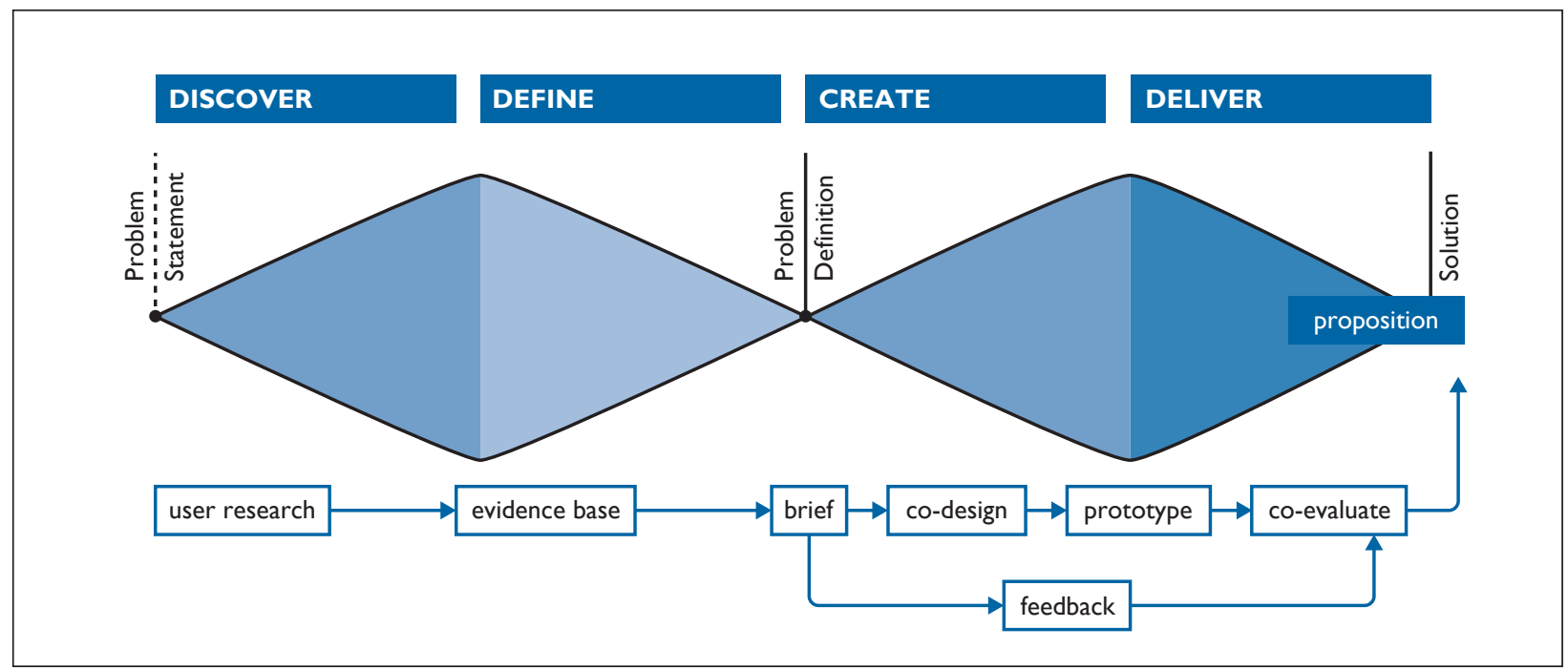

FIGURE I Combining the Inclusive Design approach with the 'Double-Diamond' Model of Development

of adult patients suffering from a wide range of medical conditions requiring urgent or emergency care, usually within 48 hours of admission or referral from other specialties. Acute hospitals are those intended for shortterm medical and/or surgical treatment and care. ${ }^{4}$

Acute medical care involves products, processes, systems and services. The design discipline is involved in creating all of these, so it should have something to offer to improve them. That is, unless the consensus is that everything in acute medical care is already perfect, in terms of quality and safety. Well, is it? Acute medicine is but a subset of medicine in general. To err is human, and a system will always fail at its weakest point. Systems are themselves designed, whether by specific intent or by evolutionary processes, and can all too easily contain imperfections. ${ }^{5}$

\section{INCLUSIVE DESIGN AND THE 'DOUBLE DIAMOND’ MODEL OF DEVELOPMENT}

The Helen Hamlyn Centre for Design is based at the Royal College of Art in London. It has developed and become a leading exponent of the Inclusive Design discipline during the last 20 years. Based on principles of people-centred research and ethnographic study, it harnesses co-creation, rapid prototyping and user evaluations to develop designs that increase access to better products and services for a wider range of people, regardless of age or disabilities. It powerfully combines its Inclusive Design methodologies with the 'Double Diamond' model of product development advanced by the UK Design Council in 2005 (Figure I). The diamond on the right hand side visualises a process of creative ideas generation, followed by systematic selection of preferred concepts for refinement, in order to deliver a working solution. Prior to that, it employs a similarly 'diamond-shaped' process to the left hand side, to build up an evidence-based design brief. This brief is derived by first establishing a clear understanding of the problem that needs to be solved, within its context or environment, through research. The research findings are then reviewed, analysed and focused, to establish a problem definition - the design brief.

The Centre employs this Inclusive methodology in many projects, including DOME (Designing Out Medical Error), ${ }^{6}$ led by its Healthcare Research Lab from 2008 to 201 I.

In up to $10 \%$ of all hospital admissions, some kind of adverse incident occurs, more than half of which are believed to be avoidable. Apart from the impact on patients (and staff), in the UK alone the consequential costs are more than $£ 2$ billion per year, and resulting media coverage damages patient trust.

DOME followed the entire patient journey through elective surgery, carrying out observations and user research in order to identify and prioritise the 'top five' ward processes (in terms of hazardousness). Observations from immersive, empathic, and ethnographic research were analysed using risk management and business theory, then comparing healthcare with other, analogous industries that routinely have to manage risk. The top five areas of error, in terms of impact on quality and safety, were poor handwashing, observations monitoring, infection control, administration of medication, and staff handover.

Design briefs were developed to address these risk areas. A co-design process of several sessions of creative ideas generation was undertaken with designers, patients, clinicians and a wide range of other stakeholders. Frontline staff contributed their expertise to establish selection criteria that were then used to rank and select the most promising solutions for further development. These were rapidly mocked up and evaluated, then refined through user feedback in an iterative process. 
The project delivered product, environmental and behaviour-influencing design interventions:

CareCentre a trolley unit that addresses all five of the top hazard areas, providing aprons, gloves, alcohol gel, a medication locker, a used sharps container, a waste bin and a proper work surface for reading and writing notes. This has been commercialised and is now being sold successfully.

Vital Signs Trolley provides self-tidying, easy to clean systems for measuring blood pressure, oxygen saturation, temperature, respiratory and heart rate, all captured presented and uploaded from a tablet screen.

Handover Area a blueprint for converting existing rooms used for staff meal breaks and rest periods into professional meeting spaces during the process of briefing the incoming team at shift changeover.

Handwashing a system of signs and sensory cues, clean zone indications and prompts to promote awareness and correct handwashing protocol.

So, what impact can Inclusive Design offer to the continuing drive to improve quality and safety in acute medical care?

There are many roles, viewpoints, skills, and demands among the different people who deliver healthcare, and co-design delivers a far greater diversity of potential solutions than professional designers alone can bring. This assures better alignment of the solutions with more of the individual and collective needs of the wide range of people who will use and interact with them.

'Design Thinking' offers several merits. It is characterised by its inherent practice of 'zooming out' for overview, and zooming in again to think at the level of detail that needs to be addressed in order to make things work. It can be incisively linear when necessary, or provocatively non-linear, and this contrast of approach is excellent for stimulating creativity. The thinking can focus on people and behaviours, as well as artefacts. Designers are intuitively biased towards solving problems, whether through product, process or systems-based solutions.

The creative process can interact with clinical research by proposing solutions that respond to the findings of research. But it can also offer provocations and propose 'What if...?' scenarios that can influence directions for future scientific research that will be needed to inform new propositions and validate development efforts.

What are the typical outputs of the Inclusive Design approach and their impact? These can be objects or products, printed information, screen-based data or images that can help to inform, provide answers or influence behavioural change. Or they can be new processes or systems. Service design propositions can be evolved from these innovations, valuable because they address whole system solutions. If well executed, this variety of interventions can lead to significant improvements in patient safety.

At the time of writing, a project is under way between the Royal College of Physicians of Edinburgh and the Helen Hamlyn Centre for Design, employing the principles outlined in this article to address the challenges of maintaining good patient flow through acute medicine. The project is moving into the Define phase, which will shortly establish the form that the design intervention will take at its conclusion at the end of September 2015.

4 Acute medical care. http://en.wikipedia.org/wiki/ Acute_\%28medicine\%29 (accessed 26/I/20I5)

5 Buckle P, Clarkson PJ, Coleman R et al. Design for Patient Safety. Department of Health, London; 2003. https://www-edc.eng.cam. ac.uk/books/bloodbag/Design\%20for\%20Patient\%20Safety_ Design_Council.pdf (accessed 26/I/20I5).

6 Anderson O, Davey G, West J. Make It Better - Designing Out Medical Error. (C) Helen Hamlyn Centre, Royal College of Art. ISBN 978-1-907342-40-0. http://www.rca.ac.uk/research-innovation/ research/current-research/make-it-better-designing-out-medicalerror/ (accessed 26/0I/20I5). 